\title{
Note à Propos des Recherches Scientifiques au Niger
}

LE Centre Nigérien de Recherches en Sciences Humaines est chargé, comme son nom l'indique, de coordonner et de faciliter la recherche fondamentale ou appliquée, dans le domaine des Sciences Humaines, sur le territoire de la république du Niger.

Afin de remplir ce rôle et de mieux servir l'ensemble de la Recherche, le Centre serait reconnaissant aux différents chercheurs projetant de travailler au Niger de lui envoyer, avant leur arrivée, les renseignements suivants:

(I) Renseignements d'ordre personnel: Nom, adresse; Emploi; Titres universitaires; Publications antérieures (le Centre aimerait recevoir un exemplaire des travaux déjà effectués joint au projet de recherche). (2) Projet de Recherches: Titre du projet; Sujet; Description détaillée; Préparation linguistique; Organisme de soutien; Projet de Budget.

Les chercheurs travaillant au Niger devront s'engager à déposer à la Bibliothèque du CNRSH un rapport provisoire à la fin de leur séjour, et devront envoyer au Centre un exemplaire au moins de leurs publications ultérieures (article, livre, thèse, dissertation) qui auront résulté de leur travail sur le terrain. Le CNRSH sera heureux d'accueillir les chercheurs étrangers, et espère que leur séjour au Niger s'avèrera profitable pour tous. D'autre part, le Centre recevra avec plaisir toutes demandes de renseignements sur la Recherche au Niger, et est prêt, dans la mesure du possible, à apporter toute son assistance aux chercheurs.

(Communication $d u$ Dr. Jean Rouch, Directeur Scientifique, I.F.A.N., Niamey, B.P. 3 I8, Tel. 3I. 4I.)

\section{L'Atlas International de l'Ouest Africain}

Le premier train de huit planches de cartes de l'Atlas International de l'Ouest Africain vient de sortir. La nouvelle ne passera pas inaperçue, tant il avait été de fois question de cette publication, désirée par tous les africanistes, mais différée sans cesse. Pour tout renseignement concernant la souscription, s'adresser à: I.F.A.N. - Atlas-B.P. 206-Dakar.

Le projet en fut en effet élaboré à l'occasion de la première Conférence des Africanistes de l'Ouest réunie à Dakar en 1945 et tout naturellement l'I.F.A.N., sous la direction de Théodore Monod, en devenait la cheville ouvrière. Des groupes de travail y furent réunis en r955 et 1956; la transformation des institutions en rapport avec l'indépendance des États africains, retarda la mise en route véritable qui n'eut lieu qu'en I960. A cette date fut établi un programme de cartes tenant compte à la fois des besoins des éventuels utilisateurs et des possibilités de la documentation. Un plan de réalisation fut mis au point en 1963 au cours d'une réunion de spécialistes tenue à l'Université d'Ibadan. Il fut approuvé comme projet $n^{\circ} 25 \mathrm{par}$ le C.S.A. devenu aujourd'hui C.S.T.R. de l'Organisation de l'Unité africaine, et il est honoré d'une subvention de la Fondation Ford qui permet de couvrir le coût du dessin et de l'impression, les frais de recherche revenant aux institutions participant aux travaux.

Le premier train comprend huit planches au format $53 \times 76 \mathrm{~cm}$. La première planche situe sur une page l'Ouest africain dans le monde au moyen de deux planisphères (climat et relief). Sur la page voisine sont présentées les mers et côtes à l'échelle la plus usuelle de $1^{\prime}$ Atlas, le $\mathrm{I} / 5.000 .000^{\circ}$. Les quatre planches suivantes sont consacrées au climat. Elles exposent ses éléments majeurs: précipitations, températures, humidité de l'air, vents au sol, d'après les données les plus significatives et notamment les moyennes 1930-60 pour la pluie. La première de ces planches se réfère aux moyennes annuelles et ses quatre cartes sont à l'échelle du $\mathrm{r} / \mathrm{r} 0.000 .000^{\circ}$. Les trois autres donnent les mêmes éléments, mais mois par mois, à l'échelle du $\mathrm{r} / 20.000 .000^{\circ}$.

Une planche au $\mathrm{I} / 5.000 .000^{\circ}$ est consacrée aux divisions politiques et administratives. Les limites des deux premières divisions à l'intérieur des États sont indiquées (si toutes deux 